\title{
Present Status of Surgical Treatment in Japan
}

\author{
Masatoshi SHIOZAWA, Vice Director \\ Research Institute Sanatorium, Japan Anti-Tuberculosis Association
}

I have the pleasure of presenting to you a report on the present status of surgical treatment.

This study has been carried out on 11,411 cases who had undergone the operation during 5 years from 1963 to 1967 at 51 institutions belonging to the Japan Tuberculosis Research Committee and had been observed postoperatively for the period from 6 months to 2 years. Based on this study, I would like to review and evaluate the present status of surgical treatment for pulmonary tuberculosis in Japan, in order to improve the result of surgical treatments in the future.

Before reviewing the study, it is considered very necessary to decide the place of surgical treatment in the treatment of tuberculosis.

According to the fourth nation-wide survey of tuberculosis prevalence carried out in 1963, the presumptive number of active cases requiring treatment is 1.5 millions, which is $1.5 \%$ of all population. Among them, the number of cases requiring surgical treatment is supposed to be 55,000 , which occupies $3.7 \%$ of the active cases. The cases of tentative chemotherapy mean that the prognosis of chemotherapy can not be decided without the result to be obtained after administration of antituberculous drugs, and these cases are 0.25 million, which corresponds to $16.5 \%$ of the active cases. They are divided into 70,000 cases with higher probability of requiring surgical treatment and 0.18 million cases with higher probability of having cure to be obtained by chemotherapy alone. Thus, the rate of cases supposed to require the surgical treatment is less than $10 \%$ of the total active cases. These numbers are very small, but they can not be expected to be cured completely by chemotherapy and to maintain much potency to the relapse after cessation of chemotherapy. Therefore, the place of surgical treatment in the treatment of tuberculosis

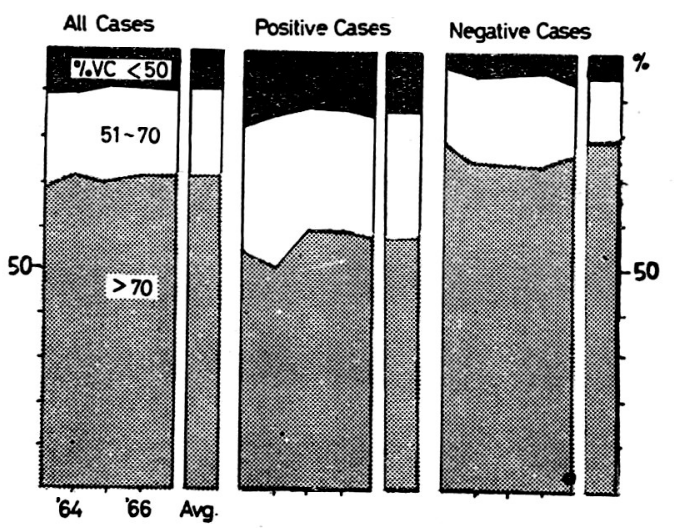

Fig. 1. Ventilatory Function Finding

Table 1.

Indication of Treatment in Tuberculosis

\begin{tabular}{l|c|c|c|c|c|c|c|c}
\hline \hline & \multicolumn{2}{|c|}{1968} & \multicolumn{2}{|c|}{1963} & \multicolumn{2}{|c}{1958} & \multicolumn{2}{|c}{1953} \\
\cline { 2 - 8 } \cline { 5 - 8 } & $\%$ & Presum No. & $\%$ & Presum No. & $\%$ & Presum No. & $\%$ & Presum No. \\
\hline Chemother. & 75.3 & 1.33 & 75.8 & 1.51 & 75.0 & 2.23 & \multirow{2}{*}{78.7} & 2.14 \\
\hline Tentative Chemother. & 16.5 & 0.25 & 15.0 & 0.23 & 18.2 & 0.40 & & \\
\hline Surgical Treatment & 3.7 & 0.06 & 4.1 & 0.03 & 1.4 & 0.04 & 7.7 & 0.12 \\
\hline Conservat. Treatment & 4.5 & 0.07 & 5.1 & 0.10 & 5.4 & 0.16 & 13.9 & 0.37 \\
\hline
\end{tabular}

(Million) 
must be considered very important in Japan.

Needless to say, it is essential to analyse the preoperative background factors of the cases operated upon and the type of surgical procedures applied, in order to evaluate the result of surgical treatments.

Observing the preoperative background factors of the cases operated upon by means of ventilatory function findings, as shown in this figure, the cases with \%VC more than 70 , that is, the favourable functional cases, are found in $70 \%$ and the cases having \%VC less than 50, namely, the lower functional cases in $10 \%$. These figures do not show a marked difference year by year, but a significant difference is observed in the preoperative bacteriological findings. The lower functional cases can be found more in the positive sputum cases than in the negative sputum cases, and the favourable functional cases reveal quite a contrary relation to the lower ones.

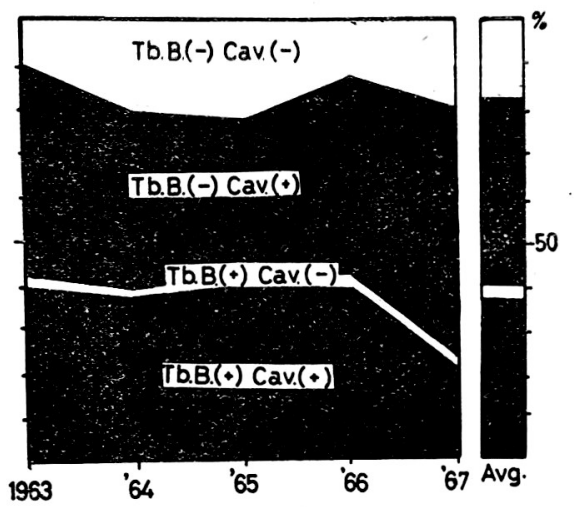

Fig. 2. Bacteriological and Radiological Findings

Dividing the cases from the standpoint of preoperative bacteriological and radiological findings, as shown in figure 3 , about $20 \%$ have negative sputum without cavity, about $40 \%$ negative sputum with cavity, about $5 \%$ positive sputum without cavity, and $35 \%$ has positive sputum with cavity. Therefore, the cavitary cases are found in $80 \%$ and the sputum positive cases in $40 \%$.

From these findings it can be said that the material includes many severe cases for the surgical treatment. However, preoperative background factors of operated cases are considered to be quite different according to countries. So, I do hope to discuss on this point later.

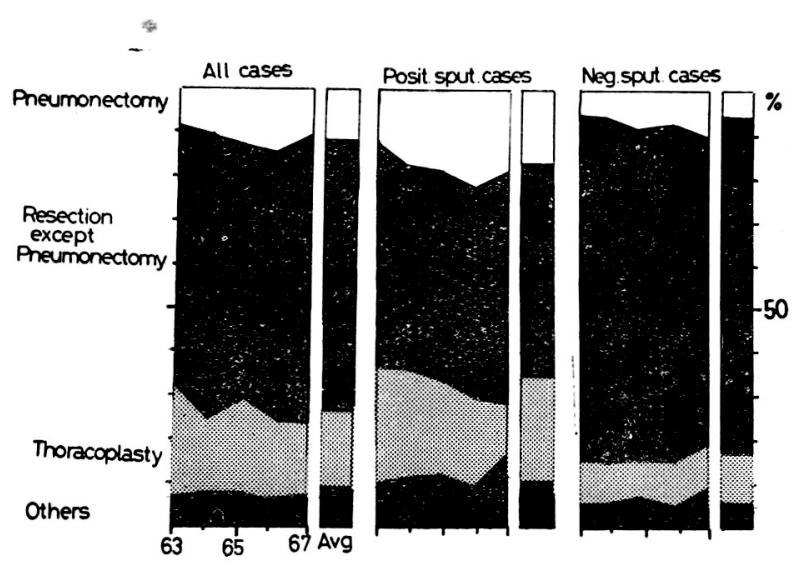

Fig. 3. Type of Operation Applied by Year

Regarding the surgical procedures applied for all cases, as shown in figure 4, pneumonectomy in more than $10 \%$, pulmonary resection except pneumonectomy about $60 \%$, thoracoplasty about $25 \%$ and other operations including cavernostomy about 5\%. Moreover, the slight increase of pulmonary resection and the slight decrease of thoracoplasty can be seen from year to year. These figures differ markedly in accordance with their preoperative background factors. For example, the rates of pneumonectomy, thoracoplasty and other operations are higher, but on the contrary, the rate of pulmonary resection except preamonectomy is lower in the positive sputum cases than in the negative sputum cases, and the slight changes of these figures are seen from year to year. The same figures are shown between the older cases and youngrr cases, and between lower functional cases and favourable functional cases.

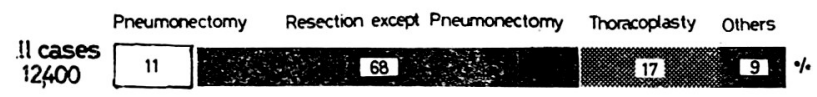

\begin{tabular}{l}
$\begin{array}{l}\text { Negative } \\
\text { Sputum }\end{array} 7$ \\
$\begin{array}{l}\text { Positive } \\
\text { Sputum }\end{array} 18$ \\
\hline
\end{tabular}

Goodcases $2\left[\begin{array}{lll}109 \\ 0\end{array}\right.$

Poor cases $26 \quad 8135$

Good cases : Tb. B. (-) and $\%$ VC> $>1$

Poor cases: Tb.B.(.) and \%VC $<50$

Fig. 4. Type of Operation Applied Preoperative Background Factors 
Figure 4 shows the frequency of surgical procedures applied in all cases, negative sputum cases, positive sputum cases, good background cases, and poor background cases.

From these findings, the tendency of the increase of pneumonectomy, thoracoplasty and other operations and of the decrease of pulmonary resecion except pneumonectcmy is seen in parallel with the increasing of the severity of disease.

I suppose the type of surgical procedures applied may differ according to each country because its choice has close relation to the preoperative background factors as well as other factors including the kind of anti-tuberculous drugs, anesthetic and operative techniques and postoperative management. Therefore, this is also one of the problems to be discussed later.

Next, I would like to analyse the result of surgical treatments by means of the success raie, the negative sputum rate, the mortality rate and the pcstoperative complication rate.

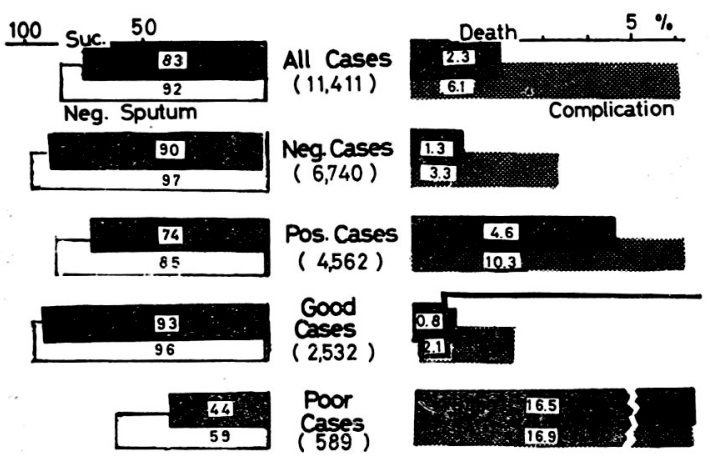

Poor case: Tb.B * , Resistance to SM\&/or INH, \%VC $<50$ andFEV $\%$ \% $\%$ C Good case : TbB-, \%VC $>81$ and FE $\nabla_{10} \%>71$

Fig. 5. Result Observed by Preoperative Background Factors

In this study the successful case is defined as the case with negative sputum who has already returned to work during the follow-up period. The mortality rate is calculatcd for the cases whose death is considered to be related to the operation and/or tuberculosis worsening postoperatively. The post-operative complications include bronchial fistula, empyema without fistula and tuberculosis worsening.

As seen in this figure, the success rate, showing red coloured column, more than $90 \%$, mortality rate, showing dark coloured column, $2.3 \%$, and the postoperative complication rate, showing green coloured column, 6. $1 \%$. However, these figures differ markedly according to the preoperative background factors. For instance, the result is significantly better in the negative sputum cases than in the positive sputum cases.

When the poor case and the good case are defined as the cases having the criteria given in the lower part of this figure the result of poor cases is very poor, as compared with the good cases, that is, the success rate is $44 \%$ the negative sputum rate $59 \%$, whereas the mortality rate is $16.5 \%$ and the postoperative complication rate is $16.9 \%$.

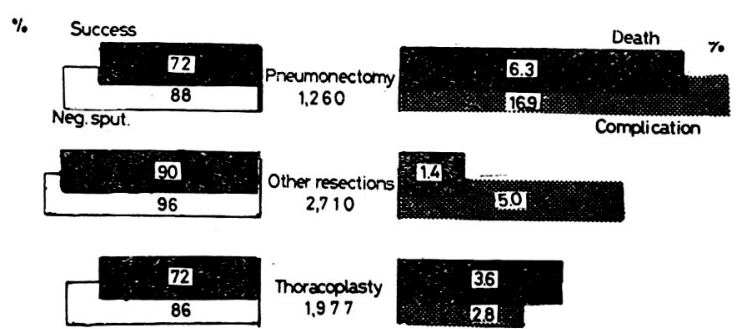

Fig. 6. Result Observed by Type of Operation

Comparing the result obtained by each surgical procedure, pulmonary resection except pneumonectomy reveals the best result, that is, the success rate is $90 \%$, the negative sputum rate $96 \%$, the mortality rate $1.4 \%$ and the postoperative complication rate $5.0 \%$ in sharp contrast to those of pneumonectomy and thoracoplasty.

Even though such results were obtained, it is not always correct to decide that lobectomy is the best method among the surgical procedures, because each procedure has a proper indication and the background factors are quite different according to surgical procedures mentioned above. under such circumstances, the comparative study of the result obtained by each surgical procedure in the cases with similar background factors, must be carried out to decide whether lobectomy is the best method or not. 


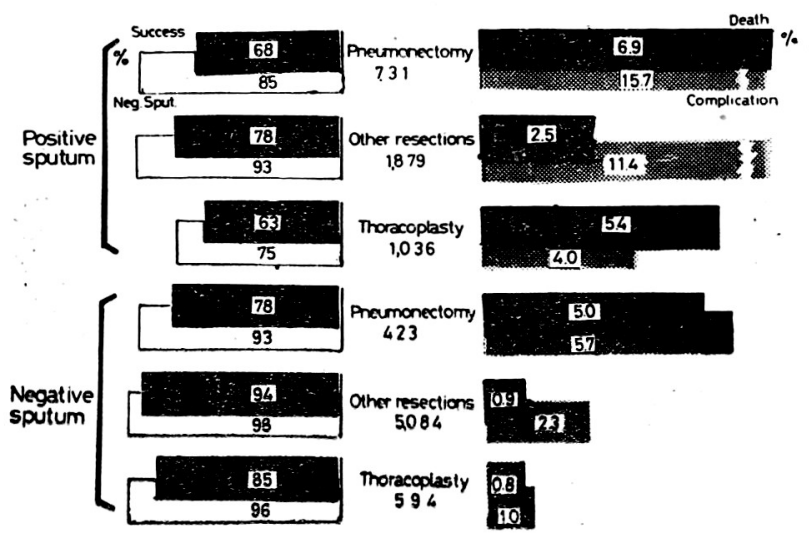

Fig. 7. Result by Type of Operation and Bacteriological Finding

As shown in this figure, in the positive sputum cases, the success rate and the negative sputum rate are higher, and the mortality rate is lower, in lobectomy than in pneumonectomy and thoracoplasty, but the postoperative complication rate in lobectomy is lower than in pneumonectomy and higher than in thoracoplasty.

In negative sputum cases, the difference of the results between three procedures becomes smaller. From these findings, the apparently good result of lobectomy is explained not by the superiority of the procedure itself, but by the preoperative favourable background factors in the operated cases.

Lastly, I must touch in brief the postoperative complication and death, in order to improve the result of surgical treatment in the future.

As shown in this figure, the incidence of postoperative complication was more than $10 \%$ for the 4 year

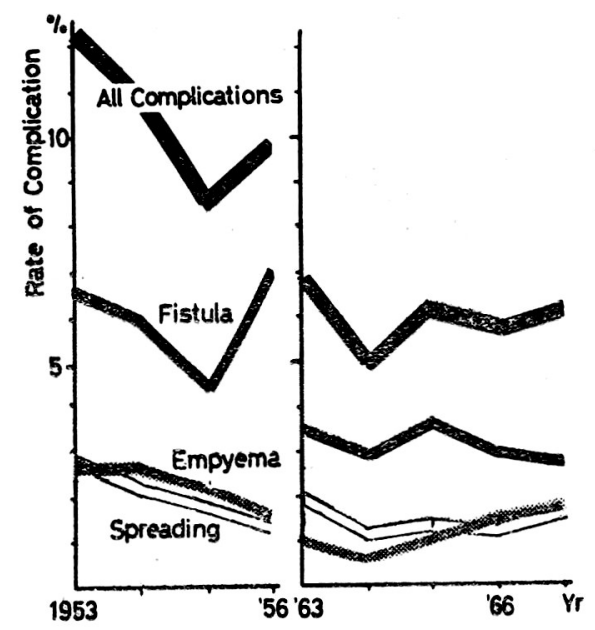

Fig. 8. Complcations of Pulmonary Resection

period about 10 years ago, but, in recent 5 years, it decreased to $6 \%$ in pulmonary resection. The postoperative complication is divided into bronchial fistula, $50 \%$, empyema without bronchial fistula, $30 \%$, and tuberculosis worsening or spread $20 \%$.

As shown in fig. 8 another trial of study makes clear that the incidence of bronchial fistula has the close relation to the duration of negative sputum before operation, positive finding of tubercle bacilli in pleural space following pulmonary resection, and the presence of histological tuberculous changes of bronchial wall at the cut surface of the bronchus. For example, the case having positive sputum immediately before operation has a high incidence of bronchial fistula, a high rate of positive tubercle bacilli in pleural space as

Table 2.

Duration of Negative Sputum and Rate of Bronchial Fistula

\begin{tabular}{|c|c|c|c|c|c|c|c|}
\hline \multirow{2}{*}{$\begin{array}{l}\text { Duration of Negative } \\
\text { sputum before opera- } \\
\text { tion (Month) }\end{array}$} & \multirow{2}{*}{$\begin{array}{l}\text { Rate of } \\
\text { bronchial } \\
\text { fistula(\%) }\end{array}$} & \multirow{2}{*}{\begin{tabular}{|c|}
$\begin{array}{c}\text { Positive* } \\
\text { tubercle } \\
\text { bacilli in } \\
\text { pleural space } \\
(\%)\end{array}$ \\
\end{tabular}} & \multicolumn{5}{|c|}{ Histalogical tuberculous changes of bronchial wall } \\
\hline & & & $\begin{array}{l}\text { Positive at } \\
\text { cut end of } \\
\text { bronchus (\% }\end{array}$ & $\begin{array}{l}\text { Main \& lobar } \\
\text { bronchus }\end{array}$ & $\begin{array}{l}\text { Segmental } \\
\text { bronchus }\end{array}$ & $\mid \begin{array}{c}\text { Subsegmental } \\
\text { bronchus }\end{array}$ & $\begin{array}{l}\text { Sub subseg- } \\
\text { mental } \\
\text { bronchus } \\
\end{array}$ \\
\hline 0 & 12. 7 & 45.6 & 27.8 & H & HI & H & Ht \\
\hline 1 & 6.9 & 29. 2 & 6.5 & - & H & $H$ & H \\
\hline 2 & 1.6 & 9.1 & 4.0 & - & - & H & H \\
\hline $3 \sim$ & 2.2 & 7.5 & 0 & - & - & $H$ & $H$ \\
\hline
\end{tabular}

* means positive tubercle bacilli in the fluid obtained at the time of washing pleural space after pulmonary resection. 
well as a high rate of histological tuberculous changes of the bronchial wall.

These incidences decrease markedly in the cases having 2 months' negative sputum before operation, but no further changes of their incidences can not be observed in the cases with 3 months' or lower negative sputum before operation.

According to these facts, the best effort should be made for the colversion to negative sputum or the decreasing amount of discharging bacilli before operation by means of the adequate chemotherapy or/and the preparatory operation, in order to decrease the occurence of postoperative complication especially bronchial fistula. If such conditions can not be obtained, by selecting the larger resections, such as, lobectomy and pneumonectomy, it is considered much safer as compared with the selection of smaller resections, such as, segmentectomy and partial resection.

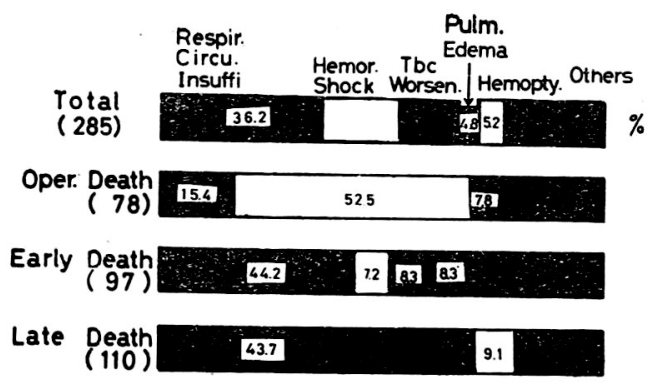

Fig. 9. Main Causes of Death

This figure shows the incidence and the main causes of deaths. The operative death is about $25 \%$, the early death about $30 \%$, and the late death about $45 \%$. However, this proportion varies according to the type of operation and the background factors.

As the cause of deaths, the respiro-circulatory insufficiency is the most predominant, about $36 \%$, next the hemorrhagic shock, $15 \%$, tuberculosis worsening, $13 \%$, pulmonary edema and hemoptysis, $5 \%$, respectively in all cases. Needless to say, that proportion differs markedly according to the type of deaths.
In other words, the hemorrhagic shock is most predominant in the operative death, whereas the respirocirculatory in ufficiency is most predominant in the early death and the late death.

In conclusion, I would like to present here the last one This figure represents the indication of surgical treatment and the selection of surgical treatment. Pulmonary resection occupies a larger part of surgical indication and thoracoplasty and other operations occupy very small part. Moreover, the latter 2 surgical procedures are overlapped in larger part by pulmonary resection.

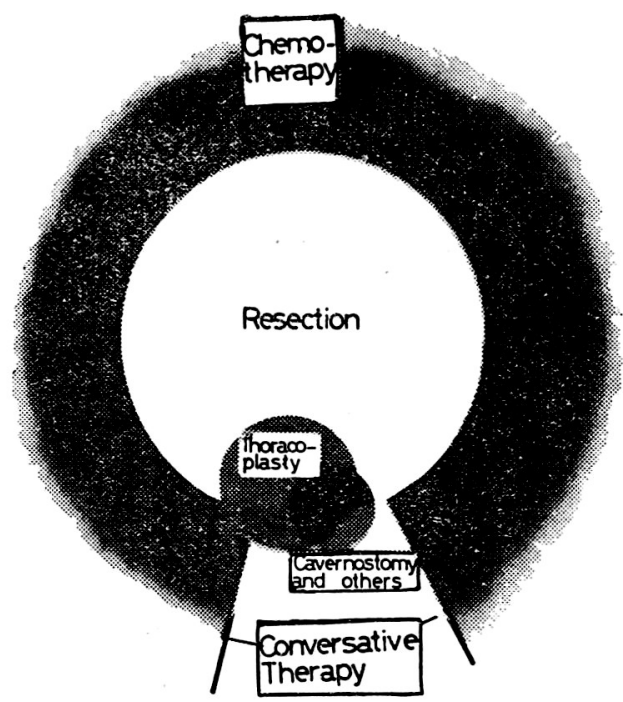

Fig. 10. Indication of Operation Method

From these facts, it can be concluded that the surgical treatment is supported by pulmonary resection in $70 \%$, by thoracoplasty $25 \%$ and other $5 \%$. The result of surgical treatment is satisfactory, and it will become better in the future by means of the improvement of operation technique as well as the improvement of management during and after operation, and more accurate determination of the indication for surgical treatments.

Thank you. 